\title{
Les premiers ingénieurs industriels issus de l'École polytechnique
}

Exemples et essai de caractérisation, $1800-1840$

The first industrial engineers from the École polytechnique: some examples and an attempt of characterization, 1800-1840

\section{Alexandre Moatti}

\section{(2) OpenEdition}

\section{Journals}

\section{Édition électronique}

URL : http://journals.openedition.org/artefact/6303

DOI : $10.4000 /$ artefact.6303

ISSN : 2606-9245

\section{Éditeur :}

Association Artefact. Techniques histoire et sciences humaines, Presses universitaires du Midi

\section{Édition imprimée}

Date de publication : 7 janvier 2021

Pagination : 77-98

ISBN : 978-2-8107-0706-5

ISSN : 2273-0753

\section{Référence électronique}

Alexandre Moatti, «Les premiers ingénieurs industriels issus de l'École polytechnique », Artefact [En ligne], 13 | 2020, mis en ligne le 23 décembre 2020, consulté le 25 décembre 2020. URL : http:// journals.openedition.org/artefact/6303 ; DOI : https://doi.org/10.4000/artefact.6303

\section{c) (i) $\ominus$}

Artefact, Techniques, histoire et sciences humaines est mise à disposition selon les termes de la Licence Creative Commons Attribution - Pas d'Utilisation Commerciale - Pas de Modification 4.0 International. 


\section{Les premiers ingénieurs industriels issus de l'École polytechnique}

Exemples et essai de caractérisation, 1800-1840

\section{Alexandre Moatti}

\section{Résumé}

Quels ont été les premiers polytechniciens industriels et comment peut-on les caractériser ? Rappelant les grandes masses agrégatives des carrières polytechniciennes dans la première moitié $d u x x^{e}$ siècle, l'article examine cinq carrières de polytechniciens pendant cette période, à dix promotions d'écart : Dufaud, Vicat, Guimet, Lafarge, Goüin. Ces ingénieurs industriels apparaissent aussi comme ingénieurs-savants, ingénieurs-inventeurs, ingénieurs-entrepreneurs et même ingénieurs-investisseurs dans des entreprises industrielles. Deux d'entre eux développent leur " innovation » au sein même des corps d'État auxquels ils appartiennent. Cet échantillon laisse entrevoir ce qu'une prosopographie des polytechniciens industriels de la période 1800-1840 apporterait à l'histoire industrielle française et à létude des relations entre État et industrie privée.

\section{Mots-clés}

industrie, grands corps, Dufaud, Goüin, Vicat, Lafarge, Guimet

95 Alexandre Moatti, « Les premiers ingénieurs industriels issus de l'École polytechnique. Exemples et essai de caractérisation, 1800-1840 », Artefact, 13, 2020, p. 77-98. 


\section{The first industrial engineers from the Ecole polytechnique: some examples and an attempt of characterization, 1800-1840}

\section{Abstract}

Who were the first industrial Polytechnicians and how can we characterize them? Recalling the great aggregative masses of polytechnic careers in the first half of the $19^{\text {th }}$ century, the article examines five careers of Polytechnicians during this period, each one with a ten-years gap between their promotions: Dufaud, Vicat, Guimet, Lafarge, Goüin. These industrial engineers also appeared as scholars, inventors, entrepreneurs, and even investors in industrial firms. Two of them developed "innovation" within the "Corps d'État" to which they belonged. This sample suggests that a prosopography of industrial Polytechnicians 1800-1840 would contribute to French industrial history, and to the study of relationships between French State and private industry.

\section{Keywords}

industry, Grands Corps of the French State, Dufaud, Goüin, Vicat, Lafarge, Guimet 


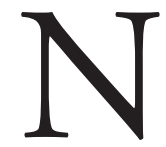

ous proposons d'examiner ici un certain nombre de carrières industrielles de polytechniciens durant une quarantaine d'années à partir de la création de l'École polytechnique en 1794. Plusieurs objectifs nous y conduisent, sur la base de l'historiographie et des travaux existants. Mais d'abord une première question se pose : pourquoi des polytechniciens? La fondation de cette école obéissait à d'autres objectifs, mais, de la même manière qu'elle a eu un impact sur la science en France entre 1800 et 1840, quel a pu être son impact sur l'industrie et comment se renouvelle, avec elle, la notion d'ingénieur en France ? Si la création de l'École centrale, en 1828, répond au souci de renforcer le lien avec l'industrie, on ne doit pas pour autant négliger l'apport à l'industrie qu'ont pu avoir ces nouveaux ingénieurs, les premiers polytechniciens. Sans prétendre répondre de manière définitive à ces questions, nous souhaitons nous inscrire dans la piste tracée par deux démarches assez indépendantes, en essayant de les croiser.

Dès 1978, l'historien Louis Bergeron s'interrogeait sur l'apparition dans le capitalisme français du début du Xix ${ }^{e}$ siècle d'un " personnage nouveau : le polytechnicien ${ }^{1}$ ", indiquant à son propos : "Simple partage de l'autorité patronale sur le plan technique ou de la gestion ? Accession au capital ? Intégration familiale par le mariage? Ce ne sont pas seulement les origines sociales et les carrières professionnelles qu'il faudrait reconstituer, mais bien les biographies individuelles ${ }^{2}$. " C'est sur la base des biographies individuelles, suivant un échantillon dont nous allons expliquer la constitution, que nous souhaitons travailler.

Quinze ans plus tard, le philosophe et historien des sciences Ivor GrattanGuinness proposait la fertile notion "d'ingénieur-savant ${ }^{3}$ ", sur cette même période 1800-1840, et soulignait le rôle déterminant de la fondation de l'École polytechnique dans l'émergence de cette catégorie, composée d'une grande majorité de polytechniciens. Elle conduit à des profils plus proches de la science que de l'industrie, mais nous examinerons si l'on peut étendre ce type de caractérisation en direction de l'industrie : peut-on parler d' "ingénieur-inventeur ", d' " ingénieur-industriel », voire d' "ingénieur-investisseur ", à propos des polytechniciens de ces mêmes années ?

\footnotetext{
1. Bergeron, 1978, p. 66.

2. Ibid., p. 68.

3. Grattan-Guinness, 1993.
} 


\section{Rappel des tendances agrégatives}

Si nous souhaitons travailler sur des biographies individuelles, comme le suggère Louis Bergeron, permettant de préciser le cas échéant une typologie d'ingénieurs (de type Grattan-Guinness), il importe néanmoins de rappeler les grandes tendances agrégatives, telles que les ont décrites d'autres auteurs. Elles restent des tendances : il n'existe pas à notre connaissance d'exploitation quantitative des carrières (inconnues pour la grande majorité) des deux cents polytechniciens de chacune des quarante premières promotions.

André Grelon fait remarquer que la grande tendance des polytechniciens industriels, dans la première moitié du XIx ${ }^{\mathrm{e}}$ siècle, est avant tout celle des ingénieurs des industries militaires de l'État, par exemple, les poudreries ${ }^{4}$. En ce sens, s'est incarnée la volonté du révolutionnaire et ingénieur-savant Lazare Carnot de former un encadrement en soutien à l'armée révolutionnaire (artillerie, génie, etc.).

Bruno Belhoste a pour sa part esquissé une classification des polytechniciens " industriels ", qui, "sans exagérer les différences ", distingue ${ }^{5}$ :

1. les établissements industriels directement gérés par l'État, et plus spécialement par l'Armée (artillerie, génie, génie maritime - corps militaires) ;

2. parmi les corps civils, ceux donnant lieu à activité industrielle, Tabacs et Poudres ;

3. les établissements privés fournisseurs des premiers (Artillerie et Génie maritime) ;

4. les autres corps civils, Mines, Ponts, avec les canaux de navigation, puis surtout les compagnies ferroviaires : « le passage du service public au service privé est rendu possible par l'institution des congés à durée limitée accordés aux ingénieurs des corps civils, qui peuvent être indéfiniment renouvelés »; 5. les autres, sachant que « rares sont les polytechniciens qui réalisent de véritables carrières d'entrepreneurs ou d'ingénieurs civils [...] les patrons polytechniciens sont des exceptions avant la fin du XIX ${ }^{\mathrm{e}}$ siècle $(2,5 \%$ suivant une enquête portant sur le Second Empire) ».

4. Discussion lors de la séance du séminaire CIHE 2019, 12 septembre 2019, Paris.

5. Belhoste, 2003, sous-chapitre «Les polytechniciens et l'industrie », p. 409-412. 


\section{Sources relatives aux ingénieurs industriels polytechniciens}

Notre démarche étant d'analyser et de caractériser quelques carrières individuelles de polytechniciens dans l'industrie, à partir de la promotion 1794, comment faire un choix, et sur quelles bases? Il existe des sources qu'on peut qualifier de primaires, non des annuaires, mais des livres commémoratifs portant sur l'École polytechnique et mettant en avant un certain nombre d'individualités, comme Le Livre du Centenaire (1894) ou L'École polytechnique (1932).

\section{L’ingénieur - l'ingénieur civil}

Dans ces ouvrages, la part réservée à la rubrique "Industrie " est limitée et vient à la toute fin de ces recueils des contributions de l'École polytechnique à la nation. Ainsi en est-il de la table des matières de l'ouvrage de 1932: Sciences - Lettres - Administration publique («Hommes d'État») - Services militaires (Armée de terre - de mer) - Services civils, dont : Corps et, assez loin, Industrie. Tout se passe comme si l'industrie polytechnicienne était d'abord et avant tout celle menée par les corps d'État, avec une forme de condescendance envers les industriels non " corpsards " :

Un grand nombre d'Ingénieurs, surtout dans les Mines, les Ponts et Chaussées et le Génie maritime, ont pu, grâce à des congés illimités ou des missions, se livrer à l'Industrie sans quitter leurs Corps. [...] D'autres ont eu une vie moins facile. Amenés par des causes diverses à ne point entrer dans les administrations de l'État [...] [ils] occupent dans l'Industrie une très haute situation et rendent à la Patrie des services au moins aussi utiles que s'ils étaient restés fonctionnaires ${ }^{6}$.

Suivant cette historiographie " maison ", les polytechniciens industriels méritant d'être relevés sont donc avant tout les " corpsards ». Il y a là un souci de légitimer les corps par les services industriels rendus à la nation et ainsi de justifier le "privilège de recrutement " que Polytechnique a

6. Le Livre du Centenaire, 1894. 
vis-à-vis de ces corps ${ }^{7}$. Cette primauté des Grands corps sur l'idée même d'industrie en France est une longue histoire : comme le soulignait le sociologue Ezra Suleiman, "presque tous ceux qui parlent de l'industrie en France avec une audience nationale sont d'anciens hauts fonctionnaires ${ }^{8}$ ". De même, Terry Shinn en 1978' Antoine Picon en 1992 et Hélène Vérin en 1993 rappellent les évolutions que connaît la dénomination d'ingénieur à partir du XIX ${ }^{e}$ siècle. D'ailleurs, en 1935, le Dictionnaire de l'Académie française $e^{10}$ précise encore ce sont " ceux qui appartiennent aux Corps de l'État». A. Picon ajoute que les anciens Centraliens voulurent fonder une "Union des ingénieurs civils et industriels ${ }^{11}$ " - l’ingénieur avec épithète est forcément moins noble que l'ingénieur, lui sans épithète.

\section{Choix d'un échantillon}

Ces éléments de contexte une fois posés, la question du choix des sources pour isoler des carrières individuelles de polytechniciens demeure. Les livres commémoratifs mentionnés ci-dessus constituent une source quasi-primaire, au plus près de la période considérée. Nous y avons choisi cinq parcours, un par décennie, Dufaud X1794 (première promotion), Vicat X1804, Guimet X1813, Lafarge X1825 et Goüin X1834. C'est un travail d'échantillonnage, par définition très partiel : bien évidemment une étude plus exhaustive, portant sur un échantillon de 50 ingénieurs (10 par décennie), eût été plus significative. Mais comment les choisir, dans des listes de noms quasi tous inconnus, et surtout de la carrière desquels il ne reste quasiment pas de traces, sinon éparses et difficiles à rassembler ?

Les cinq ingénieurs ci-dessus ont déjà été étudiés individuellement ; par ailleurs ils figurent aussi dans des sources secondaires, ce qui est une forme de validation partielle du choix. D'autres choix auraient pu être faits, en complément de cet échantillon : Antoine-Rémy Polonceau (1778-1847,

7. C’est l'exclusivité (très tôt contestée) de l'École polytechnique, depuis l'article XVI, titre II, du décret du 30 vendémiaire an IV (22 octobre 1795).

8. Suleiman, 1979.

9. Shinn, 1978, qui note : « les ingénieurs des Corps de l'État refusent systématiquement - et bien souvent avec un certain mépris - de reconnaître la légitimité professionnelle et sociale des fonctions de leurs collègues du secteur privé. Le statut d'ingénieur de l'industrie s'en trouve donc amoindri $[\ldots] »$.

10. Cité par Vérin, 1993, p. 39.

11. Picon, 1992. 
X-Ponts 1796), qui fonde, en 1838, avec Marc Seguin, une société pour la construction du viaduc de Meudon ; ou, à l'autre extrémité de notre spectre de promotions, Louis Le Chatelier (1815-1873, X-Mines 1834), premier d'une prestigieuse lignée polytechnicienne ${ }^{12}$, inventeur de nouveaux procédés industriels (dans les locomotives, l'extraction de l'aluminium) lorsqu'il est au service de l'État, puis en situation de congé au Crédit mobilier des frères Pereire, de 1855 à 1868, puis de nouveau au service de l'État ${ }^{13}$. Ce sont là deux " corpsards » et ce statut mériterait une étude spécifique en regard de l'industrie - nous y reviendrons. En tout état de cause, deux autres " corpsards » (Vicat, Guimet) figurent déjà dans notre échantillon. Un autre polytechnicien industriel de la période est mentionné par Denis Woronoff ${ }^{14}$ : il s'agit de François Gracchus Cabrol (1793-1882, X1810), dirigeant des fonderies du duc Decazes à Decazeville (Aveyron), un ingénieur industriel non entrepreneur lui-même.

Sur la base de ces cinq parcours choisis dans la première moitié du XIx ${ }^{\mathrm{e}}$ siècle - avec les limites d'un tel choix explicitées ci-dessus -, nous tâcherons de renseigner les catégories mentionnées précédemment : ingénieur-savant, ingénieur-industriel, ingénieur-entrepreneur, ingénieur-financier. Au-delà, se dessinent d'autres perspectives que nous examinerons en dernière partie, la moindre n'étant pas la participation des ingénieurs polytechniciens à la fondation de l'industrie française.

\section{Cinq parcours de polytechniciens dans l'industrie}

\section{Georges Dufaud (1777-1852, X1794)}

Dufaud est peut-être l'industriel polytechnicien le plus représentatif parmi les premières promotions de l'École. Par sa date de naissance et sa filiation, il

12. À l'inverse de Louis, son fils Henry Le Chatelier (1850-1936, X-Mines 1869), scientifique de renom, a fait l'objet d'études approfondies (par exemple Letté, 2004).

13. Thépot, 1994 , p. 80 , note à propos de ce "rétro-pantouflage " : "On ne peut s'empêcher d'admirer l'extrême mansuétude de l'administration des Mines à l'égard de Louis le Chatelier qui put en fait continuer les recherches techniques qu'il avait commencées [...] l'administration des Mines montrait ainsi qu'elle savait reconnaître les services rendus au Corps, même si ceux-ci s'étaient effectués dans le privé. "

14. Woronoff, 1998 [1994], p. 271. 
fait le lien avec une industrie d'Ancien Régime, tout en étant de plain-pied avec la révolution sidérurgique de son siècle ${ }^{15}$. Il était le fils d'un maître de forges, celle de Beaumont (Nièvre) attestée dès 1480, à qui un aristocrate, Babaud de La Chaussade, dirigeant de plusieurs forges dans la Nièvre (dont Beaumont, Guérigny et Cosne $)^{16}$, l'avait cédée en 1781 . Après avoir participé à la création de plusieurs établissements de fonte, notamment à Montataire (Oise) et Grossouvre (Cher), Dufaud crée, en 1821, avec les Boigues comme commanditaires, les aciéries de Fourchambault (retour dans sa Nièvre natale), qui se spécialisent dans les pièces militaires et les ponts métalliques de chemin de fer. Selon Denis Woronoff, ces " usines de type nouveau " (Fourchambault ou Imphy dans la Nièvre, Hayange en Lorraine) marquent un " changement d'échelle radical ${ }^{17}$ ". C'est aussi dans ces mêmes aciéries de Fourchambault qu'Henri Fayol (le "Taylor français ") met au point ses théories de rationalisation (1888-1918).

Une filiation sidérurgique ascendante relie Dufaud aux aristocrates maîtres de forges d'Ancien Régime, mais il apparaît cependant comme un acteur de la nouvelle sidérurgie : "Il ne pouvait s'accommoder de la routine où se traînait alors l'industrie des forges et ne tarda pas à se frayer une route nouvelle dans laquelle il devait un jour être suivi avec un élan qui amena une révolution dans la fabrication du fer en France ${ }^{18}$. » Une filiation sidérurgique descendante l'unit à un autre polytechnicien puisqu'Émile Martin (1794-1871, X1812) - lui-même fils d'un ingénieur des Ponts et Chaussées non polytechnicien - épouse sa fille. Avec son fils, Pierre-Émile Martin (1824-1915, Mines de Paris) ${ }^{19}$, ils inventent le procédé Martin de fonte de ferrailles, utilisé de 1860 à $1980^{20}$. Émile Martin, gendre de

15. Bergeron consacre, dès 1978 (p. 68), quelques lignes à la dynastie Dufaud-Martin-Saglio à Fourchambault.

16. «L'entreprise que [La Chaussade] avait constituée, en cinq implantations, produisait des ancres et des fers pour la marine royale. Au total, une dizaine de hauts-fourneaux et une quarantaine de forges s'activaient alors, sous sa direction, pour ce seul débouché militaire. " (Woronoff, 1998 [1994], p. 29).

17. Woronoff, 1998 [1994], p. 214.

18. Saglio, 1893. Alfred Saglio (1823-1893, X1841) avait épousé la fille aînée d'Achille Dufaud, fils de Georges.

19. Comme l'écrit fort justement la Médiathèque de Nevers (bibl.) : "La personnalité d'Émile Martin et celle de son fils Pierre-Émile ne sont pas faciles à cerner, on a grand'peine à saisir dans le détail leur carrière. "

20. Encore dans les années 1980, la Société métallurgique de Normandie (groupe Usinor) produisait à partir de ferrailles avec un " convertisseur Martin ». 
Dufaud, devient aussi député de la Nièvre à la Constituante de 1848. Une autre des filles de Dufaud avait épousé George Crawshay, fils d'un maître de forges anglais ${ }^{21}$. Même si Dufaud était déjà fils d'un maître de forges, le capital social de la famille se trouva renforcé par ces mariages polytechniciens ou trans-Manche.

Comment donc classer Dufaud, cette "personnalité attachante 22 ", cet ingénieur qui, avant tout provincial, fait le pont entre la sidérurgie du $\mathrm{XVIII}^{\mathrm{e}}$ et celle du XIX ${ }^{\mathrm{e}}$ siècle ? Un entrepreneur ? Un ingénieur industriel ? Un scientifique, en disputatio avec Monge sur la composition précise et le statut de la fonte ${ }^{23}$ ? Un ingénieur-savant ( II avait une instruction théorique bien supérieure à celle de la plupart des autres maîtres de forges de cette époque ; né et élevé dans les forges, il possédait à fond la pratique du métier ${ }^{24} »$ ) ? Un inventeur (il obtient, en 1808, un brevet pour l'affinage du fer à la houille) ${ }^{25}$ ? Il est difficile de l'affecter à une catégorie : peut-être à la confluence de ces catégories, à une époque où l'on pouvait encore cumuler l'ensemble de ces caractéristiques?

\section{Louis Vicat (1786-1861, X1804)}

Deuxième ingénieur, de dix ans son cadet, Louis Vicat. Il nous amène à une autre catégorie, celle des " corpsards ». Il a été beaucoup étudié, y compris dans une histoire d'entreprise, puisque les ciments Vicat existent encore ${ }^{26}$. Dans les hommages polytechniciens, Vicat apparaît comme l'exemple du " corpsard " désintéressé, au service du bien public, ne brevetant pas son invention du ciment artificiel (1817). Il commence sa carrière comme ingénieur ordinaire des Ponts en 1809 en Dordogne, où il construit le pont de Souillac à partir de 1812 ; c'est là qu'il met au point ses découvertes : il construit une des piles du pont en ciment artificiel. Nommé

21. Saglio, 1893 , p. 11.

22. Médiathèque de Nevers Agglomération, Guides de recherche (bibl.).

23. «Convaincu par de nombreuses expériences que l'opinion de Monge, Berthollet et Vandermonde, sur l'affinage de la fonte, n'était pas exacte, il eut des discussions suivies avec le premier, qu'il réussit à convaincre. " (Saglio, 1893, p. 10). Sans doute faut-il tempérer cette assertion en tenant compte $\mathrm{du}$ caractère hagiographique de ce document d'hommage familial.

24. Saglio, 1893, p. 13.

25. Saglio, 1893, p. 11. Dufaud reçoit un prix de la Société d'encouragement pour l'industrie nationale en 1811 .

26. Le site vicat.fr propose plusieurs pages consacrées à l'histoire, ce qui n'est pas si courant sur un site internet d'entreprise. 
ingénieur en chef dans l'Isère en 1827 , « il préféra à ce service sédentaire une situation qui pût lui permettre de continuer ses recherches, et il revint à Souillac où il fut autorisé à se charger, pour le compte d'un concessionnaire, de la construction du pont d'Argentat sur la Dordogne »; " il demanda et obtint un service spécial ${ }^{27}$ ». Le concessionnaire est le comte Alexis de Noailles (1783-1835), président du Conseil général et député de la Corrèze jusqu'en 1831, qui fait construire le pont métallique suspendu d'Argentat ${ }^{28}$ pour transporter le charbon de sa mine. Les intrications entre fonctions politiques et industrielles semblaient assez lâches, en parallèle des intrications entre hauts fonctionnaires et industriels ou maîtres d'œuvre, comme le montre l'exemple de Vicat. Ceci corrobore ce qu'écrivait André Thépot à propos du corps des Mines : «Le cas [de deux ingénieurs] montre à quel point, au début de la Restauration, la distinction n'était pas encore très bien faite entre service public et industrie privée ${ }^{29}$ ». Revenu au service public, en 1839, Vicat " refusa le grade d'Inspecteur divisionnaire qui lui aurait imposé des obligations nuisibles à ses travaux "; "il refusa cette promotion qui eût exigé une résidence à Paris ${ }^{30} »$.

Aux côtés de ce service des Ponts par intermittence, Vicat a une intense production scientifique à l'Académie des sciences, dont il est nommé membre correspondant en 1833, et aux Annales des Ponts et Chaussées, menant des controverses (avec Prony et Girard à l'Académie notamment). Sur ce plan scientifique, Antoine Picon indique que Vicat était " moins confiant que Navier dans les promesses des mathématiques et de la mécanique », qu'il "insiste sur l'expérience ", qu'il « se montre plus proche de l'état d'esprit pragmatique dont font preuve les Seguin ${ }^{31}$ ». D'ailleurs Vicat se réclame de Seguin dans son ouvrage sur Argentat : "Ces observations

27. Merceron-Vicat, 1901. Comme Martin pour Dufaud, Merceron-Vicat est polytechnicien et gendre de Vicat. Nous remercions Mme C. Masteau, de la bibliothèque de l'École des ponts, de nous avoir mentionné cette référence.

28. Vicat consacre un ouvrage à cette réalisation en 1830 (Vicat, 1830). Dans cet ouvrage, la coopération avec le concessionnaire Noailles est décrite de manière tout à fait lissée ; tandis que le dossier d'archives de Vicat (Corps des Ponts, AN, F/14/2337/2) montre une controverse entre Vicat et Noailles, ainsi qu'avec l'administration des Ponts. Cette controverse pourrait être étudiée plus avant.

29. Thépot, 1994, p. 319.

30. Renaud, 1962, p. 129.

31. Picon, 1992, p. 385 
m’ont paru offrir quelque intérêt, et je me décide à les publier, à l'exemple de M. Seguin aîné ».

Alors, quelle catégorie choisir pour Vicat ? Tout se passe comme si, au sein du corps des Ponts, il n'avait pas pris dès le départ une carrière essentiellement scientifique, à la différence de Navier, et avait dû batailler tout au long de son parcours professionnel avec son corps, avec ses collègues scientifiques et ceux des Ponts pour imposer ses découvertes, et avec ses supérieurs administratifs pour obtenir d'y consacrer du temps. Alors, " ingénieur industriel »? Non. Vicat ne crée pas d'entreprise ; c'est son fils Joseph Vicat (1821-1902, X1841) qui crée l'usine-mère du Vif (Isère) ${ }^{32}$. On pourrait dire "ingénieur-inventeur ", proche dans cette catégorie d'un Marc Seguin, qui n'était pas polytechnicien. Mais on peut aussi le rattacher à celle des « ingénieurs-savants »: sa création du concept d'hydraulicité, sa discussion sur le sujet peuvent être assimilées à la création de « toute une science nouvelle ${ }^{33} »$.

\section{Jean-Baptiste Guimet (1795-1871, X1813)}

Dix ans plus tard, notre troisième exemple est celui de Jean-Baptiste Guimet. Isérois comme Vicat, il est fils d'un ingénieur des Ponts (non polytechnicien) et entre au corps des Poudres et Salpêtres en 1817. Commissaire-adjoint des Poudres dans diverses affectations provinciales, il épouse, en 1825, Rosalie Bidauld, fille de peintre et peintre de son état. Est-ce en raison de cette alliance ou de sa bonne connaissance des poudres ? Toujours est-il, qu'il concourt, en 1828, à un prix de la Société d'encouragement pour l'industrie nationale et propose une poudre de synthèse pour le colorant bleu outremer, en remplacement du coûteux lapis-lazuli. Son invention, qui est primée, prendra le nom de "bleu Guimet». Parallèlement, affecté à la poudrerie de Lyon en 1830, il est nommé par l'administration, en décembre 1832, directeur de la poudrerie et de la raffinerie de Toulouse. Après quelques mois en poste, il se décide à quitter l'administration en juillet 1834, afin de créer une usine exploitant son procédé

32. Aucun mot de cela dans la rubrique Vicat des polytechniciens célèbres, comme si la création d'une entreprise était chose accessoire, presqu'indigne de figurer dans cet étalage des services rendus à la Patrie.

33. Mary, 1862, p. 190. 
de colorant à Fleurieu-sur-Saône (Rhône), qui fut un grand succès ; son beau-frère Louis Bidauld (X1826) assure la direction d'usine ${ }^{34}$.

Ayant fait fortune, Guimet devient un investisseur industriel : il fait partie des actionnaires lyonnais fondateurs de la Société de navigation mixte à Marseille, en $1850^{35}$; il investit, en 1855 , dans les sociétés de l'ingénieur centralien Henry Merle (1825-1877) qui achète des marais salants en Camargue pour la fabrication du carbonate de soude et surtout établit à Alès (Gard) l'une des premières usines d'aluminium, la Compagnie des produits chimiques d'Alais et de Camargue Henry Merle et $\mathrm{C}^{\mathrm{ie}}$, qui deviendra Péchiney ${ }^{36}$. Le nom de Guimet est resté célèbre grâce à son fils Émile (1836-1918) qui fit fructifier l'usine paternelle et fut un mécène des arts - deux musées, l'un parisien et l'autre lyonnais, portent son nom.

"Corpsard » comme Vicat, ingénieur-inventeur comme lui, Guimet saute le pas à 40 ans pour exploiter industriellement son procédé, alors que, chez les Vicat, c'est le fils qui le fait. Ainsi, comme Dufaud, Guimet peut-il être aussi classé comme ingénieur-industriel (exploitant une usine), et plus tard, surtout, entrepreneur (investisseur dans d'autres entreprises industrielles), voire financier.

\section{Léon Pavin de Lafarge (1806-1877, X1825)}

La décennie suivante nous amène à Léon Pavin de Lafarge, du nom d'un groupe lui aussi cimentier, devenu beaucoup plus important que le groupe Vicat. La famille est aristocratique ${ }^{37}$; elle vit de ses terres et de ses rentes, mais le père Auguste Pavin de Lafarge (1777-1850) est amené, pour faire vivre sa famille de cinq enfants, à devenir, en 1816, receveur principal des

34. Tarlier, p. 51. La succession confiée par Guimet à son fils en 1860 provoque « une brouille avec Louis Bidauld, son beau-frère polytechnicien et associé de trente ans » (Tarlier, p. 97).

35. Mulsant, p. 298. Cette entreprise a été connue sous le nom de Compagnie de navigation mixte, exploitant des navires jusqu'en 1981, puis transformée en holding de participations et intégrée au groupe Paribas.

36. Merle, " qui ne disposait pas de capitaux personnels importants, avait fait appel à des souscripteurs essentiellement lyonnais " (Joly, 2013, p. 68) ; "l'un d'entre eux se détache nettement : [...] Jean-Baptiste Guimet ( (ibid., p. 83). Guimet souscrit en septembre 1855 quasi $30 \%$ des actions émises : "Cette position prédominante au capital lui vaut de prendre la présidence du conseil de surveillance, qu'il conserve jusqu’à sa mort en 1871. " Joly suit la " dynastie " Guimet jusqu’à l'arrière-petit-fils Jacques Guimet (1908-1989), qui dirige l'entreprise familiale de 1945 à sa vente en 1967, et est par ailleurs administrateur (actionnaire) de Péchiney de 1951 à 1971.

37. « Pavin de Lafarge : Poitou, Vivarais ; secrétaire du Roi 1732 » (Valette, 1977). 
contributions indirectes à Lyon ${ }^{38}$. Le fils aîné, Léon, sort de l'École polytechnique en 1829, comme officier d'artillerie, dans l'ancienne tradition aristocratique. La famille est profondément légitimiste ${ }^{39}$ : le père abandonne son poste lyonnais en 1830, à la suite de "l'usurpation de LouisPhilippe $^{40}$ " et les trois fils officiers, dont Léon, quittent l'Armée en 1832, " en soutien à la duchesse de Berry ${ }^{41}$ ». La famille reprend alors l'exploitation des carrières de chaux en sa propriété du Teil, en Ardèche, qu'elle avait affermées à un chaufournier. Léon demande " à $[s]$ on père de s'occuper de l'usine à chaux, qui ne se composait alors que de deux petits fours de $3 \mathrm{~m} 50$ à $4 \mathrm{~m}$ de haut [...] "; " et voilà Léon industriel !", s'étonne a posteriori son jeune frère dans ses mémoires - il faut lire cette interjection au prisme d'une vision que l'aristocratie avait d'elle-même : n'étaient les circonstances, sans doute était-ce déroger que passer d'officier à industriel.

La société est active, elle se développe et fournit du ciment pour les ponts de Lyon, pour l'arsenal de Toulon (1834). Cependant, en 1839, après avoir construit cinq nouveaux fours pour faire face à cette demande, Léon laisse la direction qu'il a assumée pendant sept ans à son frère Édouard. Léon avait perdu sa femme en 1838 et était entré dans une phase pieuse ${ }^{42}$. Travailler n'est pas une nécessité chez les Lafarge, cet héritage peut expliquer ce retrait, de même qu'une forme de dilettantisme. À l'inverse, la société connaissant des difficultés en 1848, en raison des "troubles ", Léon, qui en tant qu'aîné disposait peut-être d'une rente d'héritage plus importante, vient à la rescousse de son frère Édouard, en constituant la société en nom collectif L. et E. Pavin de Lafarge ${ }^{43}$. Lors de son second mariage, en 1854, il fait, avec son épouse Hélène de Rivoles, un " passage à l'usine sous des arcs de triomphe, au milieu des ouvriers rangés sur deux rangs "; " [il] était très aimé des ouvriers ${ }^{44}$ ». Léon meurt en 1877 , mais « la famille garde un

38. Une source de première main est l'ouvrage écrit par le dernier de la fratrie, qui est associé à son frère aîné dans l'entreprise et la conduira : É. Pavin de Lafarge, 1890.

39. Comme l'indique Dubois, 1998, p. 28, les Pavin de Lafarge sont des légitimistes persévérants : "Édouard [NB : frère de Léon] a été le représentant pour l'Ardèche du comte de Chambord, l'héritier du trône, et à la mort de celui-ci en 1883, on a fermé les usines en signe de deuil. »

40. Pavin de Lafarge, 1890, p. 42.

41. Ibid.

42. Pavin de Lafarge, 1890.

43. Dubois, 1998, p. 22.

44. Pavin de Lafarge, 1890, pour les deux citations. 
contrôle dominant voire majoritaire ${ }^{45}$ " jusqu'au lendemain de la Seconde Guerre mondiale. Signalons enfin que Léon est le coauteur, avec son frère Édouard, de trois mémoires techniques sur les chaux, imprimés en 1861, 1869 et $1878^{46}$.

\section{Ernest Goüin (1815-1885, X1834)}

Notre dernier exemple sera, dix promotions plus tard, celui d'Ernest Goüin. Il a été plus étudié, en même temps que sa dynastie qui s'est distinguée depuis les Ateliers de construction des Batignolles fondés en 1846 par Ernest, alors âgé de 31 ans, jusqu'à l'entreprise SPIE-Batignolles, à la Fondation Goüin et à l'abbaye de Royaumont cédée à l'État par la famille dans les années 1970. C'est une dynastie industrielle (et polytechnicienne) qu'il a fondée, ou plutôt qu'il a perpétuée, car lui-même était issu d'une dynastie déjà centenaire de banquiers membres de la très haute bourgeoisie tourangelle.

Sa fiche de sortie de l'École polytechnique indique qu'il « refus[e] d'entrer dans le Corps royal d'État-major, où il avait été classé, d'après son rang sur la liste de mérite ${ }^{47}$. " Créé en 1818, ce corps préfigurait l'école d'Étatmajor. Il n'y avait sans doute guère d'intérêt pour un jeune diplômé, issu d'une famille très fortunée, à entrer dans la carrière militaire, fût-elle celle d'officiers distingués et instruits que représentait ce corps. Démissionnaire, "il préféra devenir externe aux Ponts et Chaussées ${ }^{48}$ ", entre 1836 et 1838. C'est là sans doute que la formation à la révolution industrielle du chemin de fer est la plus adéquate. Il fait, en 1838-1840, le voyage en Angleterre, " instrument du rattrapage technologique ${ }^{49}$ ", comme Dufaud quarante ans plus tôt pour la fonte. Plus précisément, il travaille aux établissements

45. Joly, 2013, p. 88. C'est après la Seconde Guerre mondiale que « les souscriptions se multiplient [...] pour financer une forte croissance qui renforce une position de leader dans la branche cimentière $[\ldots]$ ». Joly note que le Conseil reste toutefois « sous forte influence familiale (8 héritiers sur 14 en 1919, 7 sur 12 en 1939 et même 9 sur 12 en 1949) [...] »; " ce n'est qu'en 1966 que l'équilibre entre héritiers (5) et personnalités extérieures (7) s'inverse »; " le contrôle familial a duré plus longtemps au conseil qu'au capital » (p. 89).

46. Pavin de Lafarge, 1861, 1869 et 1878.

47. Archives de l'École polytechnique, Goüin, Ernest, Alexandre, $\mathrm{n}^{\circ}$ d'immatriculation 3219 (base de données des anciens élèves, en ligne : http://bibli.polytechnique. fr/F/?func=file\&file_name=find-b\&local_base=BCXC2).

48. Park-Barjot, 2000.

49. Park-Barjot, 2006. 
Sharp à Stockville et à Liverpool : "Son rôle consistait à contrôler l'exécution des locomotives commandées à ces ateliers par la Compagnie de chemin de fer de Paris à Orléans ${ }^{50}$ ", qui est l'employeur du jeune Goüin.

De retour en France, il est, de 1840 à 1846, ingénieur traction puis chef des ateliers de fabrication à la Compagnie de chemin de fer Paris à SaintGermain, créée en 1835 et inaugurée en 1837. Il contracte un mariage lié à la fois à sa fortune de grand bourgeois et à son prestige de polytechnicien :

Par son mariage avec Anne-Mathilde Rodrigues-Henriquès, descendante d'une grande famille israélite portugaise et fille d'un administrateur de la Compagnie des chemins de fer de l'Ouest, il devint le neveu d'Émile Pereire et le beau-frère de Gustave d'Eichtal. Assuré de ces puissants appuis, Ernest Goüin décida de fonder sa propre entreprise de construction de locomotives ${ }^{51}$.

Son cas ouvre sans doute vers une période de l'histoire des polytechniciens industriels : celle qui s'appuie sur le capital (et Goüin va mobiliser celui de sa famille, de sa belle-famille et des grandes banques), dans le chemin de fer et les constructions ferroviaires (les Batignolles) notamment.

\section{Essai de catégorisation}

Nous pouvons à présent dresser le tableau suivant, faisant intervenir les différentes catégories évoquées ; plus la case est teintée (le blanc, puis quatre niveaux de gris), plus la catégorie en question s'applique à l'ingénieur concerné. 
Tableau 1. - Essai de catégorisation des ingénieurs polytechniciens

\begin{tabular}{|c|c|c|c|c|c|}
\hline & & Ingénieur & & & \\
\hline & $\begin{array}{l}\text {-financier } \\
\text { (investisseur } \\
\text { dans d'autres } \\
\text { entreprises) }\end{array}$ & -entrepreneur & -industriel & -inventeur & -savant \\
\hline $\begin{array}{l}\text { Dufaud } \\
\text { X1794 }\end{array}$ & & & & $\begin{array}{l}\text { Affinage de } \\
\text { la houille }\end{array}$ & \\
\hline $\begin{array}{l}\text { Vicat } \\
\text { X1804 }\end{array}$ & & & & $\begin{array}{l}\text { Ciment } \\
\text { artificiel }\end{array}$ & \\
\hline $\begin{array}{l}\text { Guimet } \\
\text { X1813 }\end{array}$ & & & & $\begin{array}{l}\text { Bleu outre- } \\
\text { mer }\end{array}$ & \\
\hline $\begin{array}{l}\text { Pavin } \\
\text { de Lafarge } \\
\text { X1825 }\end{array}$ & & & & & \\
\hline $\begin{array}{l}\text { Goüin } \\
\text { X1834 }\end{array}$ & & & & & \\
\hline
\end{tabular}

Quelles grandes lignes directrices peut-on inférer de ce tableau ? Le cas de Vicat, fortement déporté vers la droite, est particulier : en tant qu'ingénieur des Ponts restant au corps pendant quasiment toute sa carrière, il conserve un tropisme qui le pousse vers la science et c'est son fils qui crée l'entreprise cimentière, sur la base des procédés inventés par le père.

Ce cas mis à part, on notera que tous sont des entrepreneurs, c'est-à-dire des industriels qui, à un moment de leur carrière, sont à leur propre compte ou associés. En tant qu'entrepreneurs, ils sont capables de mobiliser des fonds d'autres investisseurs, de Dufaud (avec les Boigues) jusqu'à Goüin, avec, dans ce cas, un soutien de la grande banque parisienne. L'évolution perceptible au travers de ces cinq portraits, sur une période de quarante ans, montre aussi comment le soutien financier à l'industrie s'est institutionnalisé, entre Dufaud en 1810 et Goüin en 1845. Inversement, on voit aussi comment ces industriels savent, dès le début, associer des capitaux à leurs entreprises. Si l'on se place à gauche du tableau, on voit que, parmi ces entrepreneurs, tous sauf Lafarge (l'entrepreneur aristocrate retiré sur ses terres) ont investi dans d'autres entreprises, notamment Guimet à Alès. Enfin, si l'on revient sur la droite du tableau, quasiment tous ces ingénieurs peuvent 
être considérés peu ou prou comme des ingénieurs-savants, puisqu'ils ont écrit au moins un mémoire scientifique relatif à leur activités2

\section{Perspectives de recherche possibles}

En complément de ceux déjà entrepris, un certain nombre de travaux peuvent être entrepris à partir de ce matériau des premiers polytechniciens industriels. Matériau qui montre l'importance que ceux-ci ont pu avoir dans l'essor industriel de la France entre 1800 et 1840, en étant à l'origine d'entreprises industrielles existant pour la plupart encore de nos jours $^{53}$. La question " quelle est la place des ingénieurs polytechniciens dans le développement de l'industrie française dans la première moitié du XIX ${ }^{\mathrm{e}}$ siècle ? " nous paraît devoir être creusée, en particulier par la multiplication de biographies de personnalités, à l'image de ce que nous avons fait ici. Les apports de ces études pourraient être croisés avec l'histoire économique du pays, son histoire industrielle, celle du rôle de l'État, de ses élites, tous ces domaines se nourrissant les uns des autres. Plusieurs pistes de travail complémentaires peuvent être envisagées.

D’abord, soulignons la spécificité de cette période 1800-1840 : si l'École centrale est créée en 1828 pour former des ingénieurs industriels dont on estimait que l'École polytechnique n'en formait pas assez, ou les formait mal, ou qu'ils étaient trop liés à l'État, ceci exclut-il pour autant que l'École polytechnique en ait produits ? Nos exemples, et d'autres que l'on aurait pu détailler, montrent qu'elle en a formés et que ceux-ci ont créé des entreprises d'envergure. Une exploitation plus systématique, si elle est possible, des carrières des polytechniciens des quarante premières promotions dans l'industrie apporterait aussi des éléments ${ }^{54}$ : peut-on trouver des profils

52. Bien qu'ayant un profil plus financier, Goüin, est l'auteur, en 1845 , juste avant la création des Batignolles, avec son camarade de promotion L. Le Chatelier, au profil plus scientifique, d'un mémoire d'une cinquantaine de pages avec planches, « Recherches expérimentales sur les machines locomotives ». Seul Guimet semble ne compter aucun écrit à son actif, sauf son discours de réception, en 1849, à l'Académie de Lyon, qui n'est pas un mémoire scientifique.

53. Sur l'échantillon des cinq industriels, on trouve encore quatre entreprises existantes, la sidérurgie de Dufaud étant plus difficile à tracer.

54. Comme l'indique Dhombres, 1987 (p. 16) à propos des polytechniciens : "L'évolution des profils des carrières, en particulier dans les corps où les archives sont facilement disponibles et les traditions relativement cataloguées, comme par exemple le système du pantouflage, n’a semble-t-il fait l'objet d'aucune étude systématique ». 
d'individus qui, sans avoir été eux-mêmes entrepreneurs, jouèrent un rôle important dans le développement d'entreprises industrielles ${ }^{55}$ ?

Une deuxième piste concerne plus spécifiquement les corps d'État. Bien évidemment des analyses existent. À partir de l'exemple de la mine de La Bérardière (Loire), André Thépot a désigné comme « le rêve d'une industrie d'État au XIX ${ }^{\mathrm{e}}$ siècle " celui de " créer des industries dont [les ingénieurs des Mines] auraient la gestion et qu'ils pourraient ériger en usines modèles $^{56}$ ». De nombreux travaux existent aussi sur le chemin de fer, par exemple l'importance de Paulin Talabot (1799-1885, X-Ponts 1819) dans la création du PLM. Mais deux de nos exemples (Vicat, Guimet) permettent d'apporter des éléments d'investigation possibles à cette piste : quel est le statut des ingénieurs d'État " prêtés " à l'industrie ? À quoi correspond le "service spécial " (Vicat) ? Comment est-il accordé ? Comment, le cas échéant, se passe le retour dans l'administration ? D'autres dossiers de "corpsards", non détaillés ci-dessus, montrent que la relation avec l'administration n'est pas toujours fluide (par exemple, pour Polonceau, X-Ponts 1796) ${ }^{57}$. Louis Le Chatelier (X-Mines 1834) est sans doute l'un des premiers cas de rétro-pantouflage (départ dans l'industrie, retour dans l'administration), permettant d'éclairer assez à l'amont les relations entre État (Grands corps) et industrie. Ces notions de " pantouflage » et "rétro-pantouflage ", apparues plus tardivement dans l'historiographie des $\operatorname{corps}^{58}$, trouvent leur source ici et méritent une étude plus approfondie à partir de cas individuels.

Enfin, une prosopographie plus poussée autour des premiers polytechniciens industriels, des années 1800-1840, pourrait s'attacher à divers éléments de leur environnement socio-économique, comme les réseaux de financement de cette industrie, ses commanditaires, le lien qu'entretiennent ces ingénieurs industriels avec eux, ou encore le rôle des fratries et des descendants (fils ou gendres), et la façon dont l'École polytechnique

55. Ainsi de Cabrol, X1810, dirigeant des fonderies du duc Decazes, voir ci-dessus.

56. Thépot, 1994, p. 339.

57. Le dossier de Polonceau du corps des Ponts (AN, F/14/2302/2) montre de multiples échanges entre l'ingénieur et son administration, celle-ci estimant qu'il mêle ses activités publique et privée (avec Marc Seguin), et finissant par le mettre d'office en situation de réserve du corps.

58. On peut citer de nombreux travaux sur ces sujets : Birnbaum, 1977 ; Rouban, 2010, Joly, 2015. 
est par la suite utilisée par ces dynasties ${ }^{59}$; mais aussi l'analyse plus précise des procédés découverts et mis en œuvre par ces ingénieurs, permettant d'illustrer lors de cette période la notion d'innovation et son rapport à la science à ce moment.

Ce travail sur la typologie des ingénieurs - de l'ingénieur-financier à l'ingénieur-savant - allant de pair avec un croisement entre d'une part les biographes individuelles, d'autre part l'histoire générale de l'industrie au $\mathrm{XIX}^{\mathrm{e}}$ siècle, permettrait d'appréhender plus finement cette dernière, mais aussi l'histoire scientifique et technique du pays (par exemple, sur le thème de l'innovation industrielle), comme l'histoire politique de la place de l'État dans l'économie française (avec les débuts du pantouflage).

\section{Sources}

Médiathèque de Nevers-Agglomération :

- Guide de recherches « Georges Dufaud (1777-1852), le créateur de Fourchambault (Nièvre)», en ligne : http://mediatheque.ville-nevers. fr/54-espace-francois-miterrand/ressources/124-guides-de-recherche?start=1.

- Guide de recherches «Émile Martin et Pierre-Émile Martin :

Invention de l'Acier Martin à Fourchambault (Nièvre) ", en ligne :

http://mediatheque.ville-nevers.fr/54-espace-francois-miterrand/

ressources/124-guides-de-recherche?start $=6$.

École polytechnique. Livre du Centenaire, 1794-1894, Gauthier-Villars \& fils, Paris, 1894-1897, 3 vol.

L'École polytechnique, Société des amis de l'École, Gauthier-Villars, Paris, 1932.

Mary Louis Charles, "Notice sur la vie et les travaux de M. Vicat, inspecteur général des ponts et chaussées ", Annales des ponts et chaussées. Mémoires et documents relatifs à l'art des constructions et au service de l'ingénieur, 1862, $2^{\mathrm{e}}$ semestre, p. 180-216.

Merceron-Vicat Louis, Louis Vicat, sa vie, son cuvre et ses travaux, Vve Dunod, Paris, 1901.

59. Woronoff 1998 [1994], p. 23, relève, en fin de notre période, la façon dont "les stratégies familiales des milieux les plus innovateurs orientent les héritiers vers l'acquisition d'un fort capital scientifique. Il n'est pas étonnant de voir figurer dans les promotions de l'École polytechnique, entre 1828 et 1834, un Peugeot, un Japy, un Koechlin ». Vérification faite, il s'agit d'Armand Constant Peugeot (1809-1877, X1828), Adolphe Charles Guillaume Japy (1813-1897, X1832) et Jacques Daniel André Koechlin (1814-1837, X1834). Si le dernier est mort jeune, les deux premiers ne semblent pas avoir été d'éminents dirigeants dans les entreprises franc-comtoises de leurs familles respectives. 
Mulsant Étienne Martial, " Notice sur Jean-Baptiste Guimet ", Annales de la Société linnéenne de Lyon, tome XXV, année 1878, p. 283-301.

Pavin de LAfarge Édouard, Souvenirs de famille, Delhomme et Briguet, Lyon, 1890.

Pavin de LAfarge Léon et Édouard, Notice sur l'exploitation des terres et sables réfractaires du Teil (Ardèche) et sur la fabrication des briques réfractaires de l'usine Lafarge, du Teil, Girard et Josserand, Lyon, 1861.

Pavin de Lafarge Léon et Édouard, Note sur l'emploi des chaux hydrauliques Lafarge-du-Theil, dans les travaux de l'Océan, V. Goupy, Paris, 1869.

Pavin de LAfarge Léon et Édouard, Notice sur les chaux éminemment hydrauliques de Lafarge. Ciments Portland-Lafarge, P. Dupont, Paris, 1878.

SAGLIo Alfred, Notice biographique sur Georges Dufaud, élève de la première promotion de l'École polytechnique, ingénieur-constructeur des usines de Fourchambault (1777-1852), Imprimerie Mazeron Frères, Nevers, 1893.

VICAT Louis, Description du pont suspendu construit sur la Dordogne, à Argentat, département de la Corrèze, aux frais de M. le C Alexis de Noailles, ministre d'État, aide de camp du roi, député de la Corrèze, Carilian-Goeury, Paris, 1830

\section{Bibliographie}

Belhoste Bruno, La Formation d'une technocratie : l'École polytechnique et ses élèves de la Révolution au Second Empire, Belin, Paris, 2003.

Bergeron Louis, Les Capitalistes en France (1780-1914), Gallimard, Collection Archives, Paris, 1978.

Birnbaum Pierre, Les sommets de l'État : essai sur l'élite du pouvoir en France, Seuil, Paris, 1977.

Bonnard Daisy, "Le bleu outremer : invention(s) d'un pigment ", La Revue. Musée des arts et métiers, 2006, n 46/47, p. 26-35.

Burnel Anne, "Les Goüin, une dynastie d'entrepreneurs. Histoire des dirigeants de la société des Batignolles de 1846 à 1968 ", Entreprises et histoire, 1996/2, $\mathrm{n}^{\circ} 12$, p. 75-88.

Cartier Marie, "Lafarge. De l'entreprise familiale à la multinationale (18801947) ", École nationale des chartes, thèse de doctorat, dir. D. Barjot, 2011.

Dhombres Jean, "L'École polytechnique et ses historiens ", introduction à A. Fourcy, Histoire de l'École polytechnique [1828], Belin, Paris, 1987, p. 7-69.

Dubois Léon, Lafarge-Coppée. 150 ans d'industrie : une mémoire pour demain, P. Belfond, Paris, 1998.

Grattan-Guinness Ivor, "The ingénieur savant, 1800-1830. A neglected figure in the history of French mathematics and science", Science in Context, 6/2, 1993 , p. 405-433. 
Joly Hervé, Diriger une grande entreprise au XX' siècle. L'élite industrielle française, Presses universitaires François-Rabelais, Tours, 2013.

JoLy Hervé, «Le pantouflage des hauts fonctionnaires dans les entreprises privées françaises $\left(19^{\mathrm{e}}-21^{\mathrm{e}}\right.$ siècles). Évolution de la réglementation et des pratiques", dans Engels Jens Ivo, Fahrmeir Andreas, Monier Frédéric, Dard Olivier (dir.), Krumme Touren in der Wirtschaft. Zur Geschichte ethischen Fehlverhaltens und seiner Bekämpfung, Bohlau Verlag, Frankfurt, 2015.

LetTé Michel, Henry Le Chatelier (1850-1936) ou la science appliquée à l'industrie, Presses universitaires de Rennes, Rennes, 2004.

Park-Barjot Rang-Ri, «La société de construction des Batignolles : des origines à la Première Guerre mondiale (1846-1914) : premiers résultats ", Histoire, économie et société, $19^{\mathrm{e}}$ année, $\mathrm{n}^{\circ}$ 3, 2000, p. 361-386.

PARK-BARJOT Rang-Ri, «Le voyage en Angleterre, instrument du rattrapage technologique : l'exemple d'Ernest Goüin (1838-1839) ", dans Merger Michèle (dir.), Transferts de technologies en Méditerranée, PUPS, Paris, 2006, p. 173-184.

PICon Antoine, L'Invention de l'ingénieur moderne, Presses de l'ENPC, Paris, 1992.

Renaud Bernard, "Vicat, l'homme, l'ingénieur ", Annales des Ponts et Chaussées Mémoires et documents relatifs à l'art des constructions et au service de l'ingénieur, 1962, p. 124-131.

Rouban Luc, "L'inspection générale des Finances, 1958-2008 : pantouflage et renouveau des stratégies élitaires ", Sociologies pratiques, 2010/2, n 21 , p. 19-34.

Shinn Terry, "Des Corps de l'État au secteur industriel : genèse de la profession d'ingénieur, 1750-1920 ", Revue française de sociologie, 1978, 19-1, p. 39-71.

Suleiman Ezra, Les Élites en France. Grands corps et grandes écoles, Seuil, Paris, 1979.

TARLIER Cécyl, «L'entreprise Guimet de bleu d'outremer : de Jean-Baptiste à Jean (1826-1920) ", mémoire master 2 d'histoire, université Paris-Sorbonne, 2007 (dir. D. Barjot).

TнÉPot André, Les Ingénieurs des mines du XIXe siècle. Histoire d'un corps technique d'État, t. I : 1810-1914, Éditions Eska/Institut d'histoire de l'Industrie, Paris, 1998.

Valette Régis, Catalogue de la Noblesse française contemporaine, R. Laffont, Paris, 1977.

VÉRIN Hélène, La Gloire des ingénieurs : l'intelligence technique du XVI au XVIII siècle, Albin Michel, Paris, 1993.

Woronoff Denis, Histoire de l'industrie en France. Du XVI siècle à nos jours, Seuil, Points Histoire, Paris, 1998 [1994]. 
Alexandre Moatti

\section{L'auteur}

Alexandre Moatti est ingénieur en chef des Mines, chercheur associé HDR en histoire des sciences et des techniques à l'Université de Paris (laboratoire SPHERE UMR 7219). Ses travaux portent d'une part sur l'histoire de la critique de la science et de la modernité technique à partir de 1900, d'autre part sur l'histoire des polytechniciens au début $d u x x^{e}$ siècle. Il travaille aussi sur les usages du numérique et est fondateur et éditeur des sites bibnum.education.fr et cultureGnum.fr (Canal-U/FMSH). Contact : alexandre.moatti@univ-paris-diderot.fr 